\title{
Structure de propriété et endettement des entreprises : étude empirique sur le marché français
}

\author{
Imen Latrous \\ Université du Québec à Chicoutimi
}

\section{INTRODUCTION}

\section{Dans les pays offrant une bonne protection des droits des actionnaires externes, les entreprises recourent plus au financement par émissions d'actions que par dette.}

Les entreprises souffrent-elles de problèmes de financement? Cette question a trouvé un intérêt particulier dans le contexte de la crise financière récente, qui était marquée par une crise de liquidité. En septembre 2008, la crise financière s'est intensifiée avec la chute des cours sur les marchés boursiers et la faillite de plusieurs établissements financiers, notamment celle de Lehmann Brothers. Selon plusieurs analystes, cette situation exceptionnelle a rendu les levées de capitaux plus difficiles à réaliser. En effet, le financement direct sur les marchés boursiers était quasiment inexistant et les banques sont devenues beaucoup moins prêteuses.

De nombreux travaux académiques ont essayé d'expliquer les choix de financement des entreprises. Depuis les travaux fondateurs ${ }^{1}$ sur la non pertinence de la structure du capital, plusieurs chercheurs en finance ont tenté de relâcher certaines hypothèses du marché parfait. À ce sujet, la théorie de l'arbitrage statique (Trade-off Theory) suggère l'existence d'un ratio d'endettement optimal en prenant en compte les avantages liés à la dette telles que les économies fiscales ainsi que ses différents coûts (les coûts de détresse financière ou les coûts d'agence ${ }^{2}$ ). D'autres développements considèrent l'imperfection de l'information et son asymétrie et proposent d'autres explications à la structure du capital des entreprises. Ils considèrent alors la structure financière de l'entreprise soit comme un signal envoyé vers le marché sur les bonnes perspectives de l'entreprise, soit comme une solution aux inefficiences qui caractérisent les décisions d'investissement de la firme occasionnées par l'asymétrie de l'information ${ }^{3}$. Dans ce contexte, les dirigeants se tournent d'abord vers l'autofinancement, puis vers la dette et en dernier recours vers le financement par émissions d'actions. Ce classement des sources de financement est au fondement de la théorie du financement hiérarchique (Pecking Order Theory). En se basant sur l'approche "law and finance", d'autres travaux suggèrent une explication juridique de la structure du capital des firmes. Ils signalent alors que dans les pays offrant une bonne protection des droits des actionnaires externes ${ }^{\mathrm{a}}$, les entreprises recourent plus au financement par émissions d'actions que par dette.

D'autres chercheurs ont pris en compte la structure de propriété pour mieux comprendre les choix de financement des entreprises. Ces recherches précisent que les préférences et les objectifs des dirigeants constituent des déterminants importants de la structure du capital des entreprises. Sans négliger leurs apports considérables, ces différents travaux se sont toujours référés au contexte américain caractérisé par une dispersion de l'actionnariat et par l'importance du conflit d'intérêts traditionnel opposant les dirigeants aux actionnaires ${ }^{\mathrm{b}}$.

Les entreprises caractérisées par un actionnariat concentré ont fait l'objet de peu d'études théoriques et empiriques alors même que cette structure d'actionnariat représente la forme de propriété la plus répandue dans plusieurs pays. À l'extérieur du contexte anglo-saxon, la dispersion de l'actionnariat est un mythe. Plusieurs travaux de recherche empiriques ont mis en évidence l'importance de la concentration de 
l'actionnariat dans les pays de l'Europe Continentale et de l'Asie. Dans ces pays, les entreprises sont largement dominées par des actionnaires de contrôle, souvent des familles, qui détiennent des droits de vote en excès des droits aux dividendes.

Alors que la majorité des précédentes études empiriques s'intéresse à la structure du capital des entreprises américaines qui sont caractérisées par une dispersion de l'actionnariat, ce papier s'intéresse plutôt au contexte français dont le système de gouvernance des entreprises diffère largement de celui américain. En effet, la France est l'un des pays de l'Europe Continentale où l'actionnariat est concentré et où les actionnaires de contrôle exercent une influence dominante sur l'équipe dirigeante des firmes contrôlées. L'objectif de ce papier est d'étudier l'impact de la propriété en capital des actionnaires de contrôle sur les décisions d'endettement des firmes cotées sur le marché français. En par-

\section{CADRE GÉNÉRAL D’ANALYSE}

Les études portant sur la relation entre la structure de propriété et l'endettement, menées dans le cadre de la théorie de l'agence, aboutissent à des conclusions différentes. D'un coté, des travaux obtiennent une relation positive entre le niveau d'endettement des entreprises américaines et la propriété en capital des dirigeants, qui souhaitent ainsi se soustraire à la discipline du marché de prise de contrôle. En effet, selon la théorie de l'agence, une des raisons qui pousse les dirigeants à recourir à l'endettement, comme moyen permettant d'augmenter leur pouvoir, tient au risque de leur remplacement, notamment lors d'une prise de contrôle hostile. De l'autre, des études empiriques confirment que la participation au capital des dirigeants affecte négativement le niveau d'endettement des firmes. En détenant un pourcentage important du capital, les dirigeants subissent alors un risque non diversifiable important. Dans ces conditions, ils sont incités à réduire le niveau d'endettement afin de limiter le risque de défaut de la firme. Ces résultats conflictuels orientent la recherche vers l'étude de la relation plutôt non linéaire entre la ticulier, ce travail se propose d'examiner, en France, l'effet non linéaire de la participation au capital des actionnaires de contrôle sur le niveau d'endettement des entreprises.

Des résultats intéressants découlent de cette étude menée sur un échantillon de 118 entreprises cotées à la bourse de Paris sur la période allant de 1998 à 2002. Il en ressort que le niveau d'endettement des entreprises est différent selon que la part au capital des actionnaires de contrôle est faible ou élevée.

\section{La France est l'un des pays de l'Europe Continentale où l'actionnariat est concentré et où les actionnaires de contrôle exercent une influence dominante sur l'équipe dirigeante des firmes contrôlées.}

structure de propriété et l'endettement des firmes. Selon ces modèles théoriques, pour des niveaux de détention faibles, les actionnaires de contrôle choisissent d'augmenter à la fois leur participation au capital et le niveau d'endettement. Ils développent ainsi des stratégies leur permettant de maintenir leur position dominante dans l'entreprise. Contrairement au financement par actions, la dette permet aux actionnaires de contrôle de conserver le pouvoir et de dominer plus de ressources susceptibles d'êtres détournées à leurs profits. Dans cette perspective, les actionnaires de contrôle ont alors tendance à s'enraciner et profiter d'un espace discrétionnaire important dans le choix de la stratégie de l'entreprise (la théorie de l'enracinement).

A contrario, pour des niveaux de participation élevés, la relation entre la part au capital des actionnaires de contrôle et le niveau d'endettement est négative. Une concentration élevée du capital dans les mains des actionnaires de contrôle permet habituellement de réduire les conflits d'intérêts potentiels les opposants aux actionnaires externes. En effet, dans ces condi- 
tions, les actionnaires de contrôle subiraient une fraction importante ${ }^{c}$ de la baisse de profit qui découle de leur comportement d'expropriation des actionnaires externes. Ainsi, la convergence des intérêts des différents actionnaires inciterait à moins d'opportunisme, en particulier à travers le recours à l'endettement, de la part des actionnaires dominants au détriment des actionnaires externes (la théorie d'alignement des intérêts).

\subsection{Structure de propriété et endettement de l'entreprise : un modèle théorique}

Selon la théorie de l'enracinement, les actionnaires de contrôle développent des stratégies pour maintenir et renforcer leur position, notamment via les choix de financement de l'entreprise. En se basant sur l'hypothèse d'enracinement, lorsque le pourcentage du capital détenu par les actionnaires dominants est faible, l'endettement est fortement utilisé. La dette permet, en effet, d'augmenter le pouvoir économique des actionnaires de contrôle et de contrecarrer, par conséquent, les menaces de prises de contrôle hostiles. Dans l'optique de la théorie d'alignement des intérêts, une concentration élevée du capital dans les mains des actionnaires de contrôle permet de réduire leur opportunisme aux dépens des actionnaires externes. Selon cette théorie, lorsque la participation au capital de la coalition de contrôle est importante, l'endettement est réduit. Ainsi, la montée en puissance de la coalition de contrôle se produit sans dette. Dans ces conditions, les actionnaires de contrôle sont moins amenés à utiliser l'endettement pour détourner une partie de la valeur de la firme à leur profit. Par ailleurs, ils craignent la situation de détresse financière liée à un accroissement du levier financier. En effet, les actionnaires de contrôle deviennent trop investis dans la firme à travers leur capital financier. Ils souhaitent alors limiter le risque de sous diversification de leurs portefeuilles, et ce, à travers une réduction du niveau d'endettement de la firme. En se basant sur les hypothèses d'enracinement (entrenchment effect) et d'alignement des intérêts (incentive effect) associées à l'effet de crainte de faillite, le modèle proposé suggère que la structure de propriété affecte de manières différentes le niveau d'endettement en fonction de la part du capital détenue par les actionnaires de contrôle tel qu'illustré à la figure 1).

\section{Figure 1 : Modèle théorique de l'effet de la structure de propriété sur le niveau d'endettement de}

l'entreprise

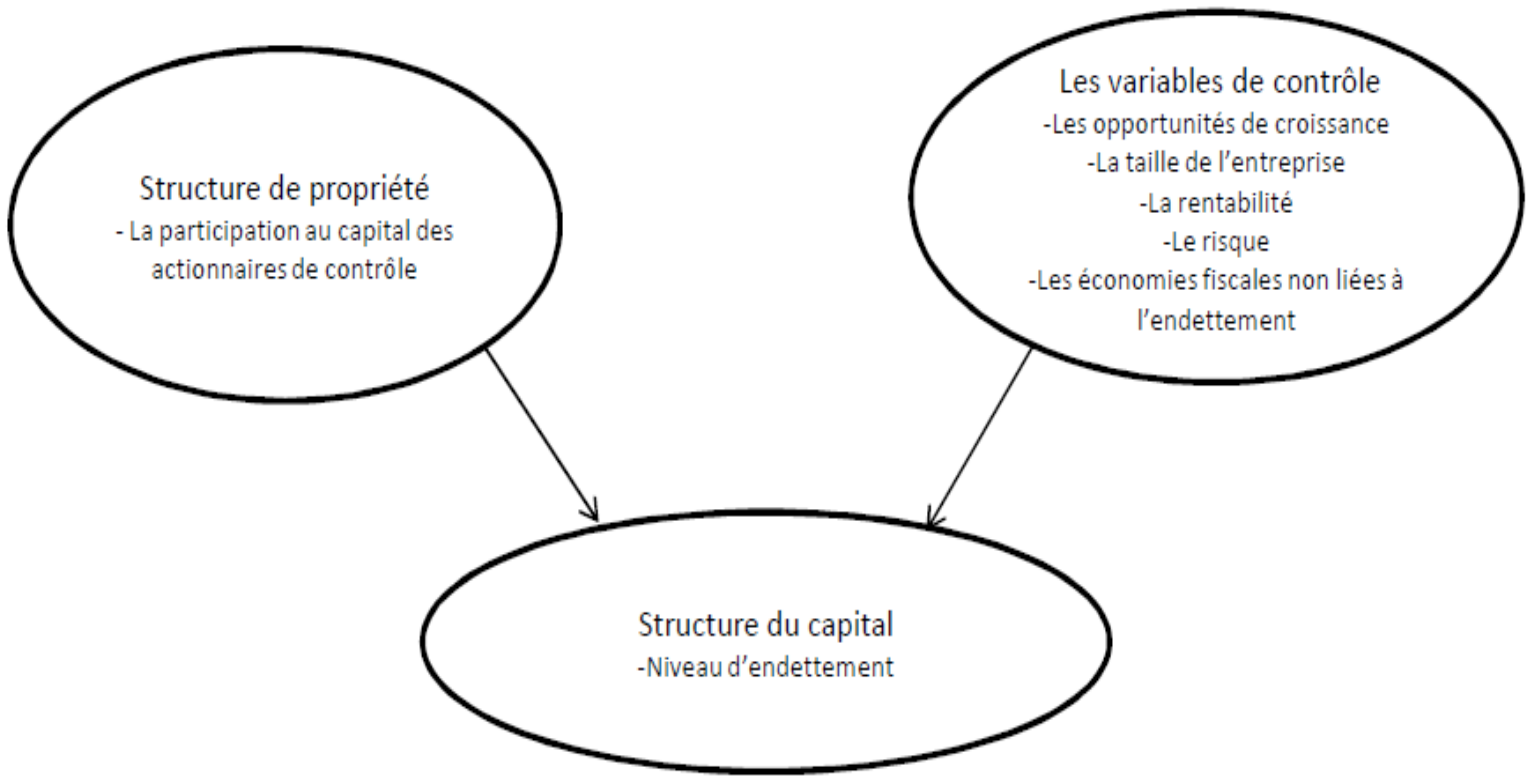




\section{MÉTHODOLOGIE}

Dans le cadre de cette étude, les données sont obtenues à partir d'un échantillon d'entreprises françaises cotées à la bourse de Paris composant l'indice SBF 250 sur la période allant de 1998 à 2002. Les institutions financières ainsi que les compagnies d'assurances ont été éliminées de l'échantillon initial à cause de leur comportement atypique en matière de politique d'endettement. L'échantillon final regroupe ainsi sur 5 ans 553 observations pour 118 entreprises sur une période de 5 ans. Le niveau d'endettement des entreprises a été calculé par le rapport de la dette financière totale rapportée à la somme de la valeur comptable des capitaux propres et de la dette financière totale (L). En outre, le plus grand actionnaire direct a été identifié afin de déterminer sa participation directe au capital et aux droits de vote. L'actionnaire de contrôle ultime est obtenu lorsque le plus grand actionnaire est une famille regroupant des individus portant le même nom ou lorsque le plus grand actionnaire est l'État. Lorsque le plus grand actionnaire est une institution financière ou une société, la chaîne de participation est alors analysée. Ainsi, la propriété en capital des actionnaires de contrôle ultimes est obtenue en prenant en compte les participations au capital tout au long de la chaîne de contrôle.
L'actionnaire de contrôle est qualifié de majoritaire lorsqu'il détient seul ou avec d'autres actionnaires liés soit par un pacte soit par des liens familiaux $40 \%$ et plus de capital ou de droits de vote $^{\mathrm{d}}$. Les entreprises sont contrôlées minoritairement lorsque le plus grand actionnaire n'est pas majoritaire au capital ou aux droits de vote au seuil de $40 \%$ mais détient avec les autres membres de la coalition de contrôle la majorité des sièges au sein du conseil d'administration. Sont membres de la coalition de contrôle autre que le principal actionnaire, le dirigeant, les actionnaires qui sont liés soit par un pacte ou soit par des liens familiaux avec le plus grand actionnaire, les banques, les assurances, l'État, les salariés non dirigeants. Lorsqu' il n'y a pas de coalition de contrôle majoritaire ni au capital ni au conseil d'administration, l'entreprise est dite alors non contrôlée (figure 2). Enfin, dans le cadre de cette analyse empirique, des variables de contrôle largement utilisées dans la littérature comme déterminants de l'endettement sont introduites tels que les opportunités de croissance, la taille de l'entreprise, la structure de l'actif, la rentabilité, le risque opérationnel, les économies fiscales non liées à l'endettement et la classification sectorielle.

\section{Figure 2 : Typologie de contrôle des entreprises}

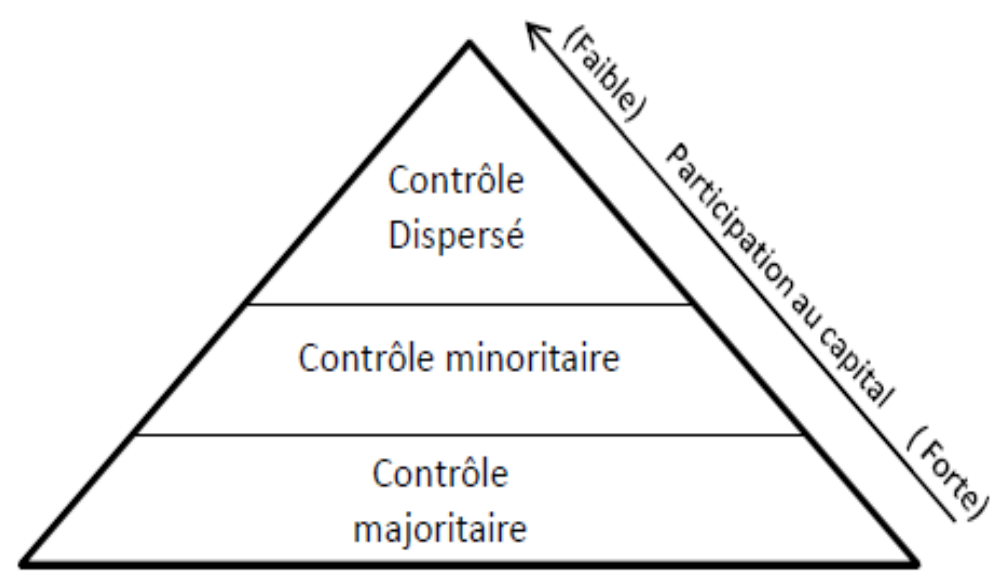




\section{RÉSULTATS}

\subsection{Caractéristiques de l'échantillon}

Les structures pyramidales et les participations croisées sont des techniques qui permettent aux actionnaires, dits de contrôle, de dominer une entreprise tout en détenant une faible part au capital.

Le tableau 1 présente la décomposition de l'échantillon total selon le type de contrôle de l'entreprise. Il est important de savoir que les actionnaires disposent de plusieurs mécanismes leur permettant de séparer la propriété (le droit aux dividendes) et le contrôle (le droit de vote) et accroître, par conséquent, leur pouvoir. Il s'agit principalement des deux classes d'actions assorties de droits de vote différenciés. Sur ce point, les structures pyramidales et les participations croisées sont des techniques qui permettent aux actionnaires, dits de contrôle, de dominer une entreprise tout en détenant une faible part au capital. Le panel A montre que $64,7 \%$ des firmes de l'échantillon sont contrôlées majoritairement, 19,9\% sont contrôlées minoritairement et seulement $15,4 \%$ des firmes ne disposent pas d'une coalition de contrôle majoritaire au capital ou au conseil d'administration. Ainsi, la décomposition de l'échantillon met en évidence la prédominance du contrôle majoritaire en France.

Tableau 1 : Répartition des entreprises par type de contrôle et par identité des actionnaires dominants

\begin{tabular}{lll}
\hline Panel A : Type de contrôle & $\begin{array}{l}\text { Nombre } \\
\text { d'observations }^{\text {e }} \\
\text { (année-entreprise) }\end{array}$ & $\begin{array}{l}\text { Fréquence } \\
(\%)\end{array}$ \\
\hline Dispersé & 85 & 15,4 \\
Contrôle minoritaire & 110 & 19,9 \\
Contrôle majoritaire & 358 & 64,7 \\
\hline Total & 553 & 100 \\
\hline
\end{tabular}

Le tableau 2 présente, pour la période allant de 1998 à 2002, les statistiques descriptives de toutes les variables utilisées dans le cadre de cette analyse empirique pour l'ensemble des entreprises de l'échantillon. En moyenne, la part de la dette financière dans l'enveloppe globale de financement est de 39,4\%. Les actionnaires de contrôle détiennent, en moyenne, 54,7 \% du capital. La taille moyenne des entreprises de l'échantillon total, mesurée par le logarithme du total bilan est de 2.81 . La rentabilité moyenne des firmes est de 7,3\% du total bilan. L'actif immobilisé moyen est de $21 \%$ et représente ainsi presque le quart du total bilan. L'écart type moyen sur 10 ans de la rentabilité, qui mesure le risque de la firme, est de 3,63\%. En moyenne, les économies fiscales non liées à l'endettement pour la totalité des firmes de l'échantillon représentent 5,2\% du total bilan.

Tableau 2 : Statistiques descriptives

\begin{tabular}{llllll}
\hline Panel A : Échantillon total & $\mathrm{N}$ & Minimum & Maximum & Moyenne & Écart type \\
\hline Le niveau d'endettement & 535 & 0.0002 & 0.994 & 0.394 & 0.220 \\
Participation au capital & 443 & 0.128 & 0.998 & 0.547 & 0.185 \\
Économies fiscales non liées à l'endettement & 551 & 0.000 & 0.660 & 0.052 & 0.042 \\
Rentabilité & 551 & -0.638 & 0.483 & 0.073 & 0.084 \\
Risque & 552 & 0.000 & 0.409 & 0.036 & 0.039 \\
Taille & 551 & 0.654 & 4.448 & 2.817 & 0.660 \\
Structure de l'actif & 551 & 0.000 & 0.966 & 0.212 & 0.173 \\
Opportunités de croissance & 549 & 0.081 & 7.942 & 2.081 & 1.443 \\
\hline
\end{tabular}




\subsection{L'effet non linéaire de la structure de propriété sur le niveau d'endettement}

Lorsque les actionnaires de contrôle détiennent des faibles parts au capital, ils cherchent alors à accroître leur pouvoir à travers un recours massif à la dette. Dans ces conditions, ils peuvent s'opposer aux menaces de prises de contrôle hostiles.

Les résultats de la recherche $\mathrm{f}^{\mathrm{f}}$ montrent que le niveau d'endettement augmente avec la participation au capital (droits aux dividendes) des actionnaires dominants jusqu'à atteindre un certain niveau au-delà ${ }^{\mathrm{g}}$ duquel l'endettement diminue avec le pourcentage du capital détenu par les actionnaires de contrôle. Lorsque les actionnaires de contrôle détiennent des faibles parts au capital, ils cherchent alors à accroître leur pouvoir à travers un recours massif à la dette. Dans ces conditions, ils peuvent s'opposer aux menaces de prises de contrôle hostiles. Dans cette situation, le recours à l'endettement est associé à l'effet d'enracinement qui devient dominant et aggravant ainsi que les conflits d'intérêts opposant les actionnaires de contrôle aux actionnaires externes. Ensuite et audelà d'un certain niveau de participation au capital, les intérêts des actionnaires dominants deviennent plus alignés sur ceux des actionnaires externes et seraient moins tentés d'augmenter le niveau d'endettement pour renforcer leur pouvoir. Dans ces conditions, l'effet d'alignement des intérêts prévaut sur l'effet d'enracinement. Par ailleurs, ce comportement limiterait le risque de défaut qui conduit à la perte de la richesse des actionnaires de contrôle. Ainsi, ces résultats corroborent la relation non linéaire entre l'endettement et le pourcentage du capital détenu par les actionnaires dominants (figure 3). En outre, les résultats de cette étude montrent que les entreprises les plus rentables utilisent significativement moins de dette que celles qui sont moins rentables.

\section{Au-delà d'un certain niveau de participation au capital, les intérêts des actionnaires dominants deviennent plus alignés sur ceux des actionnaires externes et seraient moins tentés d'augmenter le niveau d'endettement pour renforcer leur pouvoir.}

On peut donc présumer que les entreprises rentables sont peu endettées parce qu'elles se tournent plus à l'autofinancent que la dette. Un effet taille ressort, par ailleurs, des résultats de ce travail. Les entreprises de grande taille sont significativement plus endettées que celles de petite taille. Puisque ces entreprises sont principalement à maturité dont les bénéfices sont récurrents et stables, et leurs actifs sont principalement corporels, elles subissent alors un faible coût de difficultés financières : les actifs peuvent être vendus à un prix proche de leur valeur économique. Les grandes entreprises ont alors tendance à être assez endettées.

On peut donc présumer que les entreprises rentables sont peu endettées parce qu'elles se tournent plus à l'autofinancent que la dette. Un effet taille ressort, par ailleurs, des résultats de ce travail. Les entreprises de grande taille sont significativement plus endettées que celles de petite taille.

Figure 3 : Effet non linéaire de la structure de propriété sur le niveau d'endettement

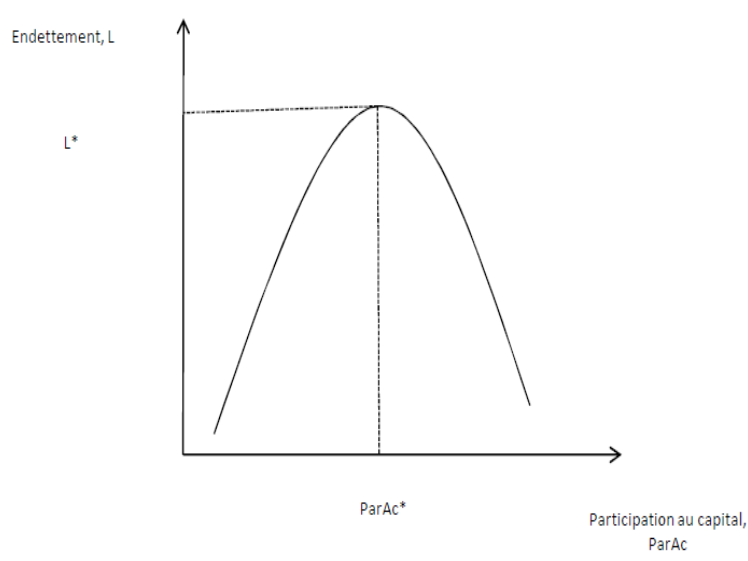

Les résultats obtenus montrent que la propriété en capital des actionnaires de contrôle affecte de manières différentes le recours à la dette. Pour des niveaux de détention faibles, l'endettement augmente avec la participation au capital des actionnaires dominants. Ce résultat s'explique pour deux raisons. D'une part, le financement par émission d'actions apporte une dilution de la propriété des actionnaires de contrôle. 
Les actionnaires préfèrent alors le

financement par dette leur permettant de renforcer leur position dominante dans

l'entreprise à travers un contrôle important d'actifs.

Les actionnaires préfèrent alors le financement par dette leur permettant de renforcer leur position dominante dans l'entreprise à travers un contrôle important d'actifs (effet d'enracinement). D'autre part, les actionnaires de contrôle minoritaires au capital craignent faiblement la faillite liée à un endettement élevé, puisqu'ils supportent une faible part de la perte de valeur si elle survient. Néanmoins, au-delà d'un niveau seuil de détention, l'endettement diminue avec la participation au capital des actionnaires de contrôle. Dans ces conditions, les objectifs de ces derniers convergent plus vers ceux des actionnaires externes. Ils seraient alors davantage concernés par la maximisation de la valeur de l'entreprise et dans une moindre mesure par l'extraction des bénéfices privés du contrôle (effet de convergence des intérêts). De plus, en investissant une part importante de leur richesse dans l'entreprise qu'ils contrôlent, les actionnaires de contrôle souhaitent sécuriser leur investissement en limitant le recours à la dette. Ils réduisent ainsi le risque de détresse financière ou l'effet de crainte de faillite. Par conséquent, la relation entre le niveau de la dette et la propriété en capital des actionnaires de contrôle est non linéaire, telle que schématisée à la figure 4.

\section{En investissant une part importante de leur richesse dans l'entreprise qu'ils contrôlent, les actionnaires de contrôle souhaitent sécuriser leur investissement en limitant le recours à la dette. Ils réduisent ainsi le risque de détresse financière ou l'effet de crainte de faillite.}

Figure 4 : Synthèse des résultats

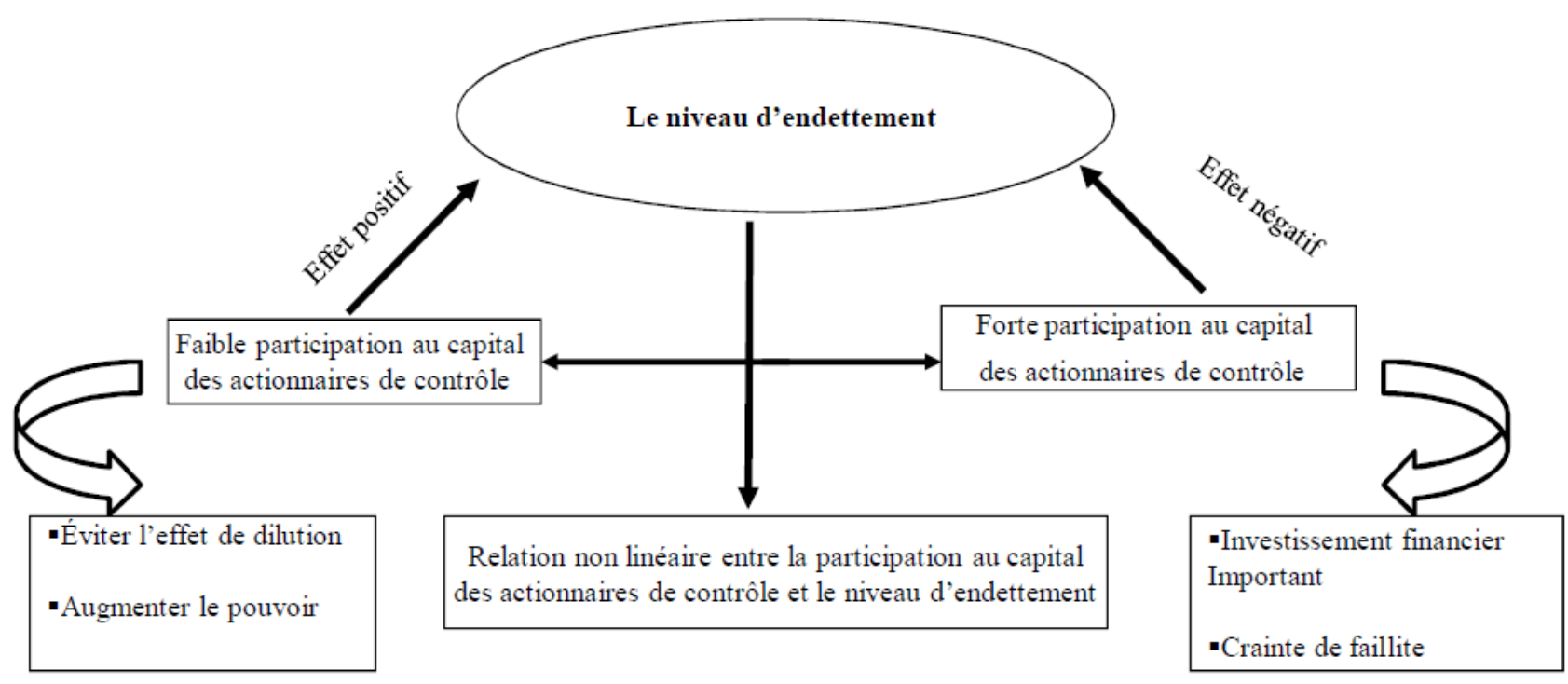




\section{CONCLUSION}

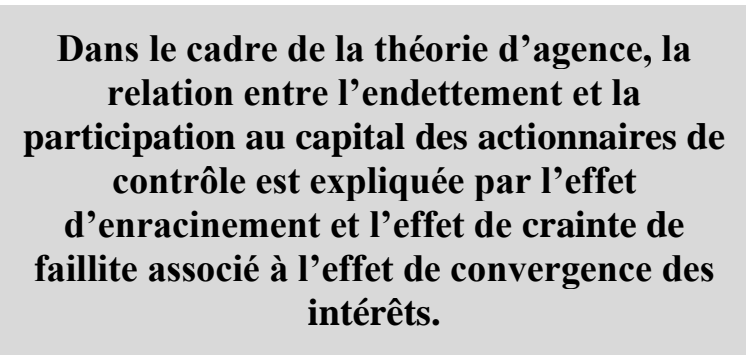

Cette étude a examiné le comportement en matière d'endettement des entreprises dominées par des actionnaires de contrôle. En particulier, nous nous sommes intéressés à la relation non linéaire entre la propriété en capital des actionnaires de contrôle et le niveau de la dette pour un échantillon de 118 entreprises françaises cotées à la bourse de Paris sur la période allant de 1998 à 2002. En inscrivant cette recherche dans le cadre de la théorie d'agence, la relation entre l'endettement et la participation au capital des actionnaires de contrôle est expliquée par l'effet d'enracinement et l'effet de crainte de faillite associé à l'effet de convergence des intérêts.

En somme, ce travail de recherche met en évidence des résultats intéressants pour les apporteurs de capitaux. Il montre que, de par leur pouvoir, les actionnaires de contrôle influencent considérablement la politique financière de l'entreprise, et plus particulièrement le choix de sa structure du capital. Cette dernière est motivée par les objectifs et les préférences propres aux actionnaires de contrôle, qui ne coïncident pas nécessairement avec ceux des actionnaires externes. Sachant que la dette permet de conserver le pouvoir et dominer plus d'actifs à exproprier par les actionnaires de contrôle, l'octroi de crédit doit être conditionné par le risque de suspendre la relation avec la banque. Ceci pousserait les actionnaires de contrôle à limiter leur comportement opportuniste au détriment des actionnaires externes.

Les actionnaires de contrôle influencent considérablement la politique financière de l'entreprise, et plus particulièrement le choix de sa structure du capital.

\section{BIBLIOGRAPHIE}

${ }^{1}$ Modigliani F., et Miller M.H., (1958). «The Cost of Capital, Corporate Finance, and the Theory of Investment », American Economic Review 48, 261-297.

${ }^{2}$ Jensen M., et Meckling W., (1976). «Theory of the Firm: Managerial Behaviour, Agency Costs, and Ownership Structure», Journal of Financial Economics, 3, 305-60.

${ }^{3}$ Myers S.C., and. Majluf N.S., (1984). «Corporate Financing and Investment Decisions when Firms Have Information that Investors Do not Have», Journal of Financial Economics, 20, 187-221.

${ }^{4}$ Brailsford T. J., Barry O.L., Pua S. L.H., (2002). «On the Relation Between Ownership Structure and Capital Structure », Accounting and Finance, 42,1-26.

${ }^{5}$ De La Bruslerie H., (2004). « Actionnaires contrôlants, bénéfices privés et endettement », document de travail, Université Paris 1 Panthéon Sorbonne.

${ }^{6}$ Faccio, M., et Lang L. H. P., (2002). «The Ultimate Ownership of Western European Corporations », Journal of Financial Economics, 65, 365-395.

${ }^{7}$ Friend I., et Lang H., (1998). « An Empirical Test of the Impact of Managerial Self-Interest on Corporate Capital Structure », Journal of Finance 43, 271-281.

${ }^{8}$ Harris M., et Raviv A., (1988). «Corporate Control Contests and Capital Structure », Journal of Financial Economics, 20, 55-86.

${ }^{9}$ La Porta R., Lopez-de-Silanes F., et Shleifer A., (1999). "Corporate Ownership Around the World», The Journal of Finance, 54, 471-518.

\section{Notes}

${ }^{a}$ Les pays du « common law ».

' $\grave{A}$ l'exception de l'approche juridique de la structure du capital qui oppose les pays de droit commun aux pays de droit civil. Toutefois, cette approche n'a pas étudié explicitement la nature de la relation entre la structure de propriété et la structure financière.

${ }^{\mathrm{c}}$ Proportionnellement à sa participation au capital.

${ }^{\mathrm{d}} \mathrm{C}^{\prime}$ est le seuil fixé par le législateur français pour définir la présomption de contrôle.

${ }^{\mathrm{e}} \mathrm{La}$ période allant de 1998 à 2002.

${ }^{\mathrm{f}}$ Les résultats détaillés sont disponibles sur demande auprès de l'auteur.

${ }^{\mathrm{g}}$ Le point d'inflexion est de $43,5 \%$ du capital détenu par les actionnaires de contrôle. 